\title{
Unacceptable failure of osteochondral glenoid allograft for biologic resurfacing of the glenoid
}

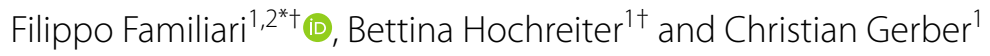

\begin{abstract}
Purpose: Glenohumeral osteoarthritis (OA) represents a challenging problem in young, physically active patients. It was the purpose of this investigation to evaluate the results of a pilot study involving glenoid resurfacing with a glenoid allograft combined with a hemiarthroplasty on the humeral side.
\end{abstract}

Methods: Between April 2011 to November 2013, 5 patients (3 men, 2 women, mean age 46.4, range 35-57) with advanced $\mathrm{OA}$ of the glenohumeral joint, were treated with a humeral head replacement combined with replacement of the glenoid surface with an osteochondral, glenoid allograft.

Results: Overall, clinically, there was one excellent, one satisfactory and three poor results. Mean preoperative subjective shoulder value (SSV) was 34\% (range: 20-50\%) and preoperative relative Constant-Murley-Score (CSr) was 43 points (range: 29-64 points). Three patients with poor results had to be revised within the first three years. Their mean pre-revision SSV and CSr were 38\% (range: 15-80\%) and 36 points (range: $7-59$ points) respectively. One patient was revised 9 years after the primary procedure with advanced glenoid erosion and pain and one patient has an ongoing satisfactory outcome without revision. Their SSVs were 60\% and 83\%, their CSr were 65 points and 91 points, 9 and 10 years after the primary procedure, respectively. Mean follow-up was 7 years (2-10 years) and mean time to revision was 4 years (range: 1-9 years).

Conclusion: The in-vivo pilot study of a previously established in-vitro technique of osteochondral glenoid allograft combined with humeral HA led to three early failures and only one really satisfactory clinical outcome which, however, was associated with advanced glenoid erosion. Osteochondral allograft glenoid resurfacing was associated with an unacceptable early failure rate and no results superior to those widely documented for HA or TSA, so that the procedure has been abandoned.

Level of evidence: Level IV, Case Series, Treatment Study.

Keywords: Shoulder osteoarthritis, young patients, osteochondral glenoid allograft, biologic resurfacing, failure rate, Constant and Murley score

\section{Introduction}

The management of the young and active patient with primary, posttraumatic or postoperative glenohumeral

\footnotetext{
*Correspondence: filippofamiliari@unicz.it

${ }^{\dagger}$ Filippo Familiari and Bettina Hochreiter are shared first authors.

2 Department of Orthopaedic and Trauma Surgery, Magna Graecia

University, Catanzaro, Italy

Full list of author information is available at the end of the article
}

osteoarthritis (OA) remains controversial. For advanced cases, hemi- (HA) and total shoulder arthroplasty (TSA) are the preferred solutions with better active mobility, pain relief, and patient satisfaction with TSA than with HA $[1,2]$. Both surgical solutions, however, are not entirely satisfactory: for TSA loosening of the glenoid component remains a too frequent cause of failure associated with revision in $0.8 \%$ of the cases per year [3-5]; 


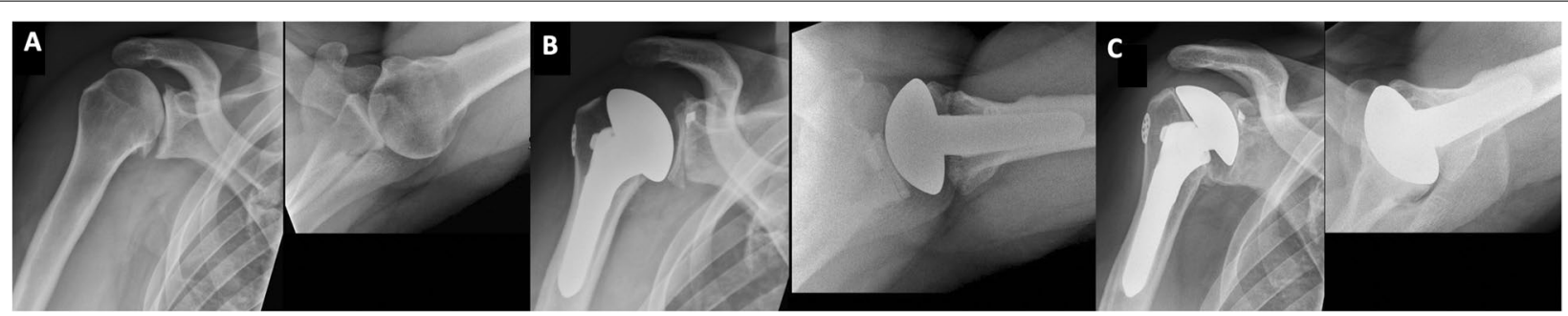

Fig. 1 Radiological FU imaging of the patient with a satisfactory course. Preoperatively (A), 1 year postoperatively (B), and 10years postoperatively (C)

and HA may be associated with progressive glenoid erosion and unsatisfactory clinical outcome in the young individual $[6,7]$.

Multiple interpositional soft-tissue grafts have been proposed to avoid or defer glenoid replacement including fascia lata, Achilles tendon allograft, anterior capsule, lateral meniscal allograft, and several dermal allografts [8-11]. Originally introduced by Burkhead and Hutton in 1995 [12], interpositional soft tissue glenoid arthroplasty, combined with HA, has been proposed for glenohumeral OA in young patients. Subsequently, conflicting results have been reported $[10,13]$. Presently, the true value of biologic resurfacing to preserve glenoid bone stock, specifically its comparison with modern TSA, remains undetermined $[11,14]$.
The concept of articular allograft replacement is well established in different fields of orthopedic surgery [15-18], with good mid- to long-term results especially in unipolar joint disease $[19,20]$. To assess the potential of glenoid allograft replacement associated with arthritic humeral head resurfacing, nine potential geometric designs of a scapular neck-glenoid allograft constructs were experimentally tested [21]. A "rectangle" design was determined to be best and was therefore chosen for five clinical pilot cases. The purpose of this clinical pilot study was to investigate the results of glenoid allograft resurfacing combined with a prosthetic humeral head replacement on the basis of positive in-vitro results and because the currently preferred options HA and TSA are not yet considered a safe and durable solution.
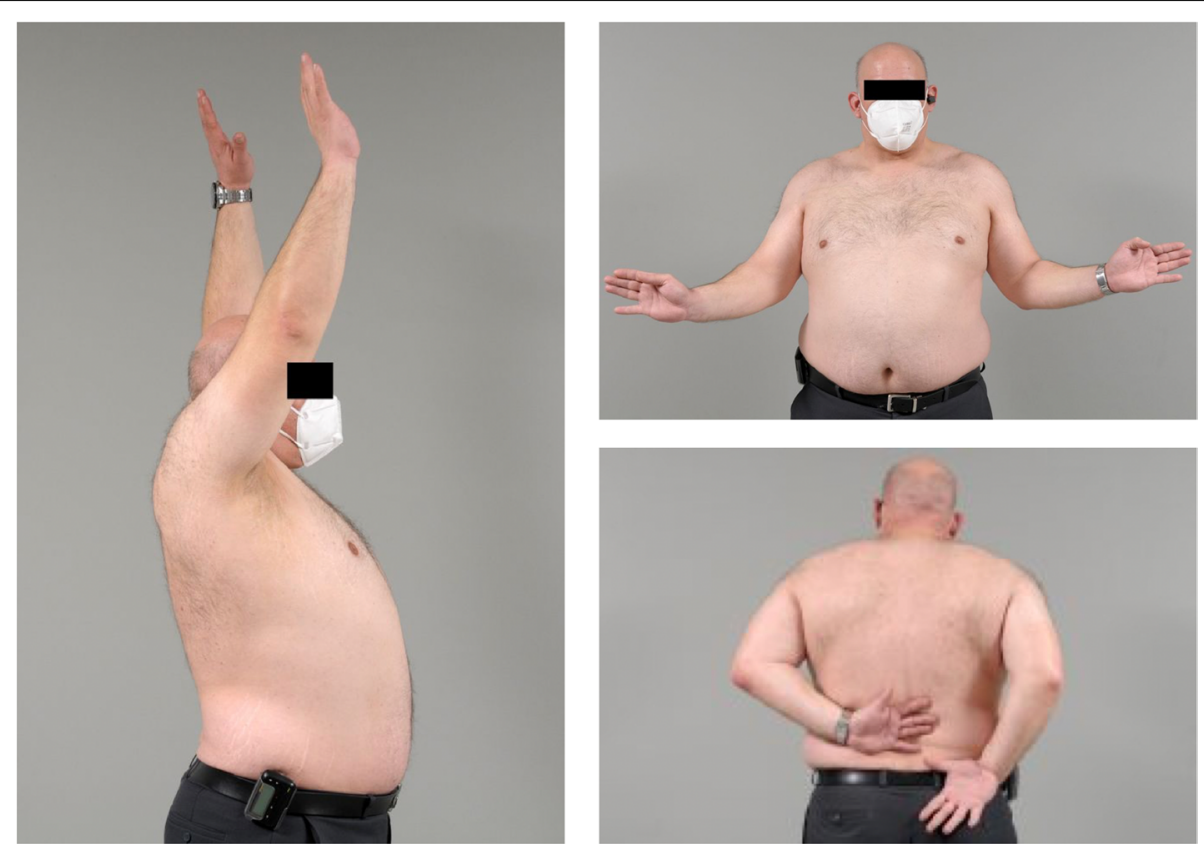

Fig. 2 Clinical imaging of the patient with a satisfactory course at 10-year FU with advanced glenoid erosion but almost unlimited clinical function 


\section{Materials and methods}

Institutional review board approval was obtained for this retrospective study (BASEC-Nr. Req-2017-00702).

\section{Study group}

From April 2011 to November 2013, 5 patients underwent a HA with an osteochondral glenoid allograft for the treatment of glenohumeral OA. The 5 patients ( 3 men and 2 women) were of a mean age of 46.4 (range, 35-57) years at surgery. The dominant arm was involved in 2 of the 5 patients.

\section{Surgical technique}

All patients received perioperative antibiotic prophylaxis. All surgeries were performed in a beach chair position under interscalene block by the same surgeon (CG). Through a deltopectoral approach the joint was approached using a lesser tuberosity osteotomy [22]. The subscapularis was mobilised, and the humeral head was exposed. A biceps tenotomy was performed routinely. The arthritic humeral head was resected anatomically after removing osteophytes. The glenoid was exposed with an anterior and posterior capsular release and the labrum was circumferentially excised. The glenoid surface was reamed flat. The size- and side matched, non arthritic, fresh-frozen glenoid allograft which had been procured from an accredited tissue bank, was prepared using a custom made cutting block. It was then slowly thawed in saline solution of body temperature. The glenoid peg hole was prepared (Fig. 1) and the definitive glenoid allograft was impacted in place (Fig. 2). The glenoid allograft was additionally stabilized with 2 anchors (Super Quick anchors; DePuy Mitek, Warsaw, IN, USA) in 1 patient. A stemmed humeral HA (Anatomical; Zimmer, Inc., Warsaw, IN, USA) was then implanted in all patients, with cementation in three and without cementation in two patients. The shoulder was reduced, and the lesser tuberosity osteotomy was anatomically repaired using transosseous sutures tied over a cortical bone plate [22].

\section{Postoperative protocol}

All patients wore a sling for 6 weeks postoperatively. For the first 6 weeks the physiotherapy protocol consisted of passive and active-assisted mobilization, with no external rotation greater than zero degrees and no internal rotation behind the back. Patients were regularly monitored at 6 weeks, 3 months, 6 months, and at 1 year after surgery or more frequently according to their clinical evolution.

\section{Patient evaluation}

All patients had complete, standardized, physical examination and scoring Subjective Shoulder Value (SSV) [23] and relative (age-adjusted) Constant-Murley (CSr) [24, 25]) as well as standardized imaging preoperatively and at final follow-up (FU) or before revision surgery.

Pre- and postoperative radiographs and computed tomographic $(\mathrm{CT})$ scans were evaluated for glenoid type according to Walch et al. [26] by 2 observers in consensus. Glenoid erosion was additionally measured on axial CT scans. For this purpose, the distance from the base of the coracoid to the glenoid articular surface was measured in the preoperative CT scans and compared with the same measurement on CT scans at latest FU or before revision.

\section{Results}

Clinically the overall results were excellent in one, good in one and poor in three cases. Mean preoperative SSV was 34\% (range: $20-50 \%$ ) and preoperative CSr was 43 points (range: 29-64 points). Three patients with a poor rating had to be revised within the first 3 years. Their mean SSV and CSr before revision were 38\% (range: 15-80\%) and 36 points (range: 7-59 points) respectively. One patient initially rated as good - had to be revised 9 years after the primary procedure. Nine years after the index procedure the SSV was $60 \%$ and CSr was 65 points. One patient has an ongoing satisfactory outcome without revision. Ten years after the index procedure the SSV is $83 \%$ and CSr is 91 points. Mean FU was 7 years (range: $2-10$ years) and mean time to revision was 4 years (range: $1-9$ years).

\section{Patients with an unsatisfactory course}

(1) The first patient, a 52-year-old woman, presented with persistent chronic pain and loss of function after sustaining a proximal humerus fracture treated with open reduction internal fixation 12 years before the index surgery. There was no major deformity of the proximal humerus but partial humeral collapse with secondary OA. According to the glenoid bone loss classification system of Walch et al. [26], the patient had a type A2 glenoid. After HA with osteochondral glenoid allograft initial pain relief and improved function were noted, but the patient worsened rapidly thereafter. The erosion of the glenoid allograft bone was $0.5 \mathrm{~cm}$ at latest FU 24 months postoperatively. Infection was ruled out and a revision to TSA was performed 2.5 years after the index procedure but did not alleviate symptoms. A revision to an RTSA was performed 9 months after the TSA and the patient was satisfied because of pain relief at latest FU with an SSV 35\% of and CSr of 43 points 5 years postoperatively. 

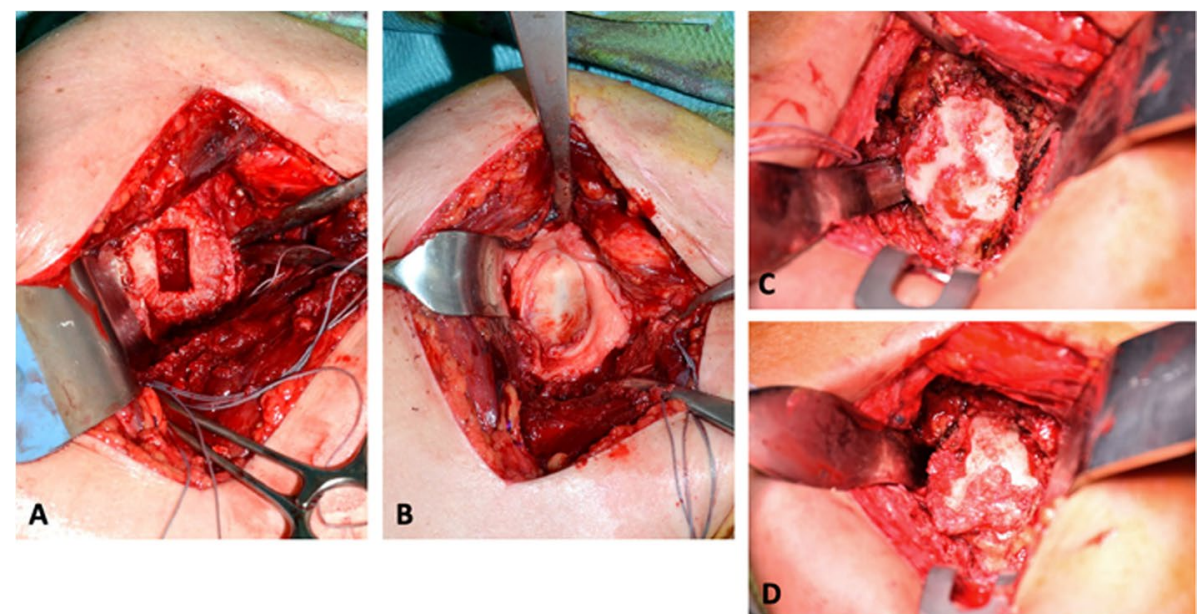

Fig. 3 Intraoperative imaging of the patient with an initially satisfactory course but revision 9 years after index procedure. Glenoid preparation at index procedure $(\mathbf{A})$, glenoid allograft after implantation (B), eroded glenoid allograft at revision surgery $(\mathbf{C}+\mathbf{D})$

(2) The second patient was a 37-year-old man who had had two stabilization procedures, including open and arthroscopic Bankart repair and subsequently developed OA (type B1 glenoid). Nine months after $\mathrm{HA}$ and glenoid allograft procedure the patient remained unacceptably painful with an SSV of $15 \%$ and a CSr of 7 points. CT showed a median erosion of the allograft of $0.3 \mathrm{~cm}$. Consecutively, conversion to an RTSA was performed 9 months after the initial procedure. At last FU, 1 year after revision surgery, the pain had clinically resolved. This patient was lost to follow-up thereafter.

(3) The third patient was a 51-year-old man who presented with primary OA (type B2 glenoid) with pain and loss of function. After HA with osteochondral glenoid allograft the pain persisted with an SSV of $20 \%$ and a CSr of 46 points. The median abrasion of the glenoid bone was $0.2 \mathrm{~cm}$ at last FU. Consecutively revision to a TSA was performed 1 year after the index procedure. Two years after glenoid conversion radiographic loosening was diagnosed. Conversion to an RTSA was offered but refused and the patient was lost to follow-up.

\section{Patients with a satisfactory course}

(1) The first patient was a 36-year-old patient with a large glenoid defect after a direct trauma (with possible posterior (sub-)luxation) who rapidly developed OA (type B2 glenoid). Nine months after HA with glenoid allograft the pain persisted during overhead and internal rotation movements as well as at night, but the patient recovered fully and presented for 10-year FU with a SSV of $83 \%$ and a CSr of 91 points (Figs. 1 and 2).

(2) The second patient was a 57-year-old woman who presented with primary OA (type A1 glenoid) with pain and loss of function. One year after HA with an osteochondral glenoid allograft she presented with persistent anterior shoulder pain especially with internal rotation but had an already improved SSV to $80 \%$ and a CSr of 59 points. Between 3 and 8 years postoperatively she had an uncomplicated and satisfactory course. Nine years after the index procedure conversion to TSA with a cemented polyethylene glenoid was performed due to a gradual increase in pain and loss of ROM. Intraoperatively bone stock was somewhat compromised, but sufficient for an anatomical restoration (Fig. 3). 4 months postoperatively the patient was very satisfied with the situation.

\section{Discussion}

The most important finding is that the technically feasible glenoid allograft for resurfacing of a degenerated glenoid surface combined with anatomical HA does not work in-vivo.

We report the results of five consecutive osteochondral glenoid allograft resurfacings of the glenoid for the treatment of glenohumeral OA young patients performed in a single institution. After unsatisfactory results with 3 other types of biological resurfacings (i.e. capsular interposition, meniscal allograft interposition, and glenohumeral GraftJacket ${ }^{\mathrm{TM}}$ interposition arthroplasty) [13], 
we aimed at trying to resurface the glenoid using a glenoid allograft. The concept was experimentally tested, the design of the construct was optimized and feasibility of glenoid allografting was validated in-vitro [15]. After successful experimental validation, a limited pilot study for patients with OA and an intact rotator cuff was set up [21]. As OA of the young can be either primary or occur after previous trauma or instability we decided to use allograft resurfacing for primary or secondary OA provided the rotator cuff was intact and there was no major posttraumatic malposition of the proximal humerus or the glenoid. At the time of this trial, the use of HA or TSA in Type B glenoids was accepted. Today, primary replacement with RTSA is preferred by many although good mid- to long-term results have also been observed with TSA [27].

A 2- to 15-year review presents the results of soft tissue glenoid resurfacing in 34 patients (36 shoulders) with a mean age of 51 years treated between 1988 and 2003. Various biologic surfaces, including anterior capsule (7 cases), autogenous fascia lata (11 cases) and Achilles tendon allograft (18 cases) were used, reflecting the evolution of the technique over time. All scores performed improved postoperatively, and using Neer's criteria, the outcome was excellent in 18 shoulders, satisfactory in 13, and insatisfactory in 5 shoulders. Unsatisfactory results were related to infection, early re-injury, and the use of the anterior capsule as interpositional material. Erosion of the glenoid averaged $7.2 \mathrm{~mm}$ but appeared to stabilize after approximately 5 years [28]. This contrasts with results of a study that published lateral meniscus allograft or human acellular dermal tissue matrix in 42 patients at an intermediateterm follow-up of 2.8 years. The lateral meniscal allograft cohort had a failure rate of $45.2 \%$, with a mean time to failure of 3.4 years. Human acellular dermal tissue matrix interposition had a failure rate of $70.0 \%$, with a mean time to failure of 2.2 years [10].

All procedures in this study were performed by a single surgeon (C.G.) using a consistent, previously laboratory tested technique. The results of 5 out of 5 patients are known and only 1 result is clinically durably good but radiographically associated with advanced glenoid erosion, a combined clinical and radiographic result well known from HA without any resurfacing [1]. A statistical analysis is not feasible as early failure with severe pain and poor function requiring revision within 3 years, one case requiring revision at 9 years and one clinically good result with an unsatisfactory radiographic course are considered prohibitive for further use of this technique. Unfortunately, no difference in clinical or radiographic parameters could be identified which differentiated the two results which were satisfactory for at least 8 years and the three poor results.

As the main limitations, the range of indications can be criticized but this corresponds to the type of OA that is seen in rather young individuals who have an intact rotator cuff. Secondly, we used commercially available, size and side matched deep-frozen allografts which were thawed in Ringer's solution at the beginning of the surgery, and we cannot exclude that some specific preparation of the graft material could have yielded better results, but the grafts used correspond to the standard used in other joints or indications. In addition, it may be criticized that there was no control group for this pilot study. The study, however, was designed to determine the safety and suitability of the procedure for a further RCT.

The early mid- and long-term results of HA and TSA for OA in relatively young patients are not yet where patients and surgeons would like them to be, but they are well established [1]. With a follow-up of 3 to 13 years, the overall implant survivorship is between 60 and $90 \%$. Glenoid loosening is the most common reason for revision (52\%). A reliable improvement of the subjective as well as objective outcome can be expected, which possibly decreases between mid- and long-term follow-up [29, 30]. Glenoid resurfacing using allograft is a theoretically attractive solution which can technically reproducibly be executed in-vitro. In-vivo, however, it led to early revision in three out of 5 cases due to severe pain, and to a result no better than the appropriately documented results of HA and TSA in the two best cases so that we have abandoned this concept.

\section{Conclusions}

We conclude that the technically feasible glenoid allograft for resurfacing of a degenerated glenoid surface combined with anatomical HA does not work in-vivo. The results in young individuals are inferior to those of $\mathrm{HA}$ and TSA.

Glenoid allograft resurfacing with prosthetic humeral head replacement is not a viable alternative for the treatment of advanced OA in the young individual due to its high risk of failure.

\section{Acknowledgements \\ None.}

Authors' contributions

Each author fulfills each of the authorship requirements. The author(s) read and approved the final manuscript.

Funding

This study received no outside funding or grants. 


\section{Declarations}

Ethics approval and consent to participate

Institutional review board approval was obtained for this retrospective study (BASEC-Nr. Req-2017-00702).

Written informed consent was obtained from all individual participants.

\section{Competing interests}

One author receives royalties from Zimmer. The other authors, their immediate families, and any research foundations with which they are affiliated have not received any financial payments or other benefits from any commercial entity related to the subject of this article.

\section{Author details}

'Department of Orthopaedics, Balgrist University Hospital, University of Zurich, Zürich, Switzerland. ${ }^{2}$ Department of Orthopaedic and Trauma Surgery, Magna Graecia University, Catanzaro, Italy.

Received: 17 September 2021 Accepted: 15 October 2021

Published online: 02 December 2021

\section{References}

1. Gartsman GM, Roddey TS, Hammerman SM (2000) Shoulder arthroplasty with or without resurfacing of the glenoid in patients who have osteoarthritis*. J Bone Joint Surg Am 82:26-34

2. Sperling JW, Cofield RH, Rowland CM (2004) Minimum fifteen-year follow-up of Neer hemiarthroplasty and total shoulder arthroplasty in patients aged fifty years or younger. J Shoulder Elb Surg 13:604-613

3. Bohsali Kl, Wirth MA, Rockwood CA (2006) Complications of total shoulder arthroplasty. J Bone Joint Surg 88:2279-2292

4. Papadonikolakis A, Neradilek MB, Matsen FA (2013) Failure of the glenoid component in anatomic total shoulder arthroplasty. J Bone Joint Surg 95:2205-2212

5. Torchia ME, Cofield RH, Settergren CR (1997) Total shoulder arthroplasty with the neer prosthesis: long-term results. J Shoulder Elb Surg 6:495-505

6. Levine WN, Djurasovic M, Glasson J-M, Pollock RG, Flatow EL, Bigliani LU (1997) Hemiarthroplasty for glenohumeral osteoarthritis: results correlated to degree of glenoid wear. J Shoulder Elb Surg 6:449-454

7. Radnay CS, Setter KJ, Chambers L, Levine WN, Bigliani LU, Ahmad CS (2007) Total shoulder replacement compared with humeral head replacement for the treatment of primary glenohumeral osteoarthritis: a systematic review. J Shoulder Elb Surg 16:396-402

8. Ball CM, Galatz LM, Yamaguchi K (2001) Meniscal allograft interposition arthroplasty for the arthritic shoulder: description of a new surgical technique. Tech Shoulder Elbow Surg 2:247-254

9. Burkhead WZ, Krishnan SG, Lin KC (2007) Biologic resurfacing of the arthritic glenohumeral joint: historical review and current applications. Shoulder Elb Surg 16:S248-S253

10. Strauss EJ, Verma NN, Salata MJ, McGill KC, Klifto C, Nicholson GP, Cole BJ, Romeo AA (2014) The high failure rate of biologic resurfacing of the glenoid in young patients with glenohumeral arthritis. J Shoulder Elb Surg 23:409-419

11. Wirth MA (2009) Humeral head arthroplasty and meniscal allograft resurfacing of the glenoid. J Bone Joint Surg 91:1109-1119

12. Burkhead WZ, Hutton KS (1993) Biologic resurfacing of the glenoid in young patients with posttraumatic arthritis, postreconstruction arthritis, or osteoarthritis. Tech Orthop 8:163-173

13. Puskas GJ, Meyer DC, Lebschi JA, Gerber C (2015) Unacceptable failure of hemiarthroplasty combined with biological glenoid resurfacing in the treatment of glenohumeral arthritis in the young. J Shoulder Elb Surg 24:1900-1907

14. Scalise JJ, lannotti JP (2008) Bone grafting severe glenoid defects in revision shoulder arthroplasty. Clin Orthop Relat Res 466:139-145

15. Elhassan B, Ozbaydar M, Higgins LD, Warner JJP (2008) Glenoid reconstruction in revision shoulder arthroplasty. Clin Orthop Relat Res 466:599-607
16. Mankin HJ, Gebhardt MC, Jennings LC, Springfield DS, Tomford WW (1996) Long-term results of allograft replacement in the management of bone tumors. Clin Orthop Relat Res 324:86-97

17. Mankin HJ, Doppelt S, Tomford W (1983) Clinical experience with allograft implantation. The first ten years. Clin Orthop Relat Res (174):69-86

18. Weng P-W, Shen H-C, Lee H-H, Wu S-S, Lee C-H (2009) Open reconstruction of large bony glenoid erosion with allogeneic bone graft for recurrent anterior shoulder dislocation. Am J Sports Med 37:1792-1797

19. Gross AE, Agnidis Z, Hutchison CR (2001) Osteochondral defects of the talus treated with fresh osteochondral allograft transplantation. Foot Ankle Int 22:385-391

20. Provencher MT, Ghodadra N, LeClere L, Solomon DJ, Romeo AA (2009) Anatomic osteochondral glenoid reconstruction for recurrent glenohumeral instability with glenoid deficiency using a distal tibia allograft. Arthroscopy 25:446-452

21. Gerber C, Snedeker JG, Krause AS, Appenzeller A, Farshad M (2011) Osteochondral glenoid allograft for biologic resurfacing of the glenoid: biomechanical comparison of novel design concepts. J Shoulder Elb Surg 20:909-916

22. Gerber C, Yian EH, Pfirrmann CAW, Zumstein MA, Werner CML (2005) Subscapularis muscle function and structure after total shoulder replacement with lesser tuberosity osteotomy and repair. J Bone Joint Surg 87:1739-1745

23. Gilbart MK, Gerber C (2007) Comparison of the subjective shoulder value and the constant score. J Shoulder Elb Surg 16:717-721

24. Constant CR, Gerber C, Emery RJH, Søjbjerg JO, Gohlke F, Boileau P (2008) A review of the constant score: modifications and guidelines for its use. J Shoulder Elb Surg 17:355-361

25. Constant CR, Murley AG (1987) A clinical method of functional assessment of the shoulder. Clin Orthop Relat Res 214:160-164

26. Walch G, Badet R, Boulahia A, Khoury A (1999) Morphologic study of the glenoid in primary glenohumeral osteoarthritis. J Arthroplast 14:756-760

27. Hendel MD, Werner BC, Camp CL, Gulotta LV, Walch G, Dines DM, Dines JS (2016) Management of the biconcave (B2) glenoid in shoulder arthroplasty: technical considerations. Am J Orthop Belle Mead NJ 45:220-227

28. Krishnan SG, Nowinski RJ, Harrison D, Burkhead WZ (2007) Humeral hemiarthroplasty with biologic resurfacing of the glenoid for glenohumeral arthritis. J Bone Joint Surg 89:727-734

29. Roberson TA, Bentley JC, Griscom JT, Kissenberth MJ, Tolan SJ, Hawkins RJ, Tokish JM (2017) Outcomes of total shoulder arthroplasty in patients younger than 65 years: a systematic review. J Shoulder Elb Surg 26:1298-1306

30. Sowa B, Bochenek M, Bülhoff M, Zeifang F, Loew M, Bruckner T, Raiss P (2017) The medium- and long-term outcome of total shoulder arthroplasty for primary glenohumeral osteoarthritis in middle-aged patients. Bone Joint J 99-B:939-943

\section{Publisher's Note}

Springer Nature remains neutral with regard to jurisdictional claims in published maps and institutional affiliations.

\section{Submit your manuscript to a SpringerOpen ${ }^{\circ}$ journal and benefit from:}

- Convenient online submission

- Rigorous peer review

- Open access: articles freely available online

- High visibility within the field

Retaining the copyright to your article

Submit your next manuscript at springeropen.com 